\title{
Eigenvalue Placement by Quantifier Elimination - the Static Output Feedback Problem
}

\author{
Klaus Röbenack ${ }^{a}$ and Rick Voßwinkel ${ }^{b}$
}

\begin{abstract}
This contribution addresses the static output feedback problem of linear time-invariant systems. This is still an area of active research, in contrast to the observer-based state feedback problem, which has been solved decades ago. We consider the formulation and solution of static output feedback design problems using quantifier elimination techniques. Stabilization, as well as more specified eigenvalue placement scenarios, are the focus of the paper.
\end{abstract}

Keywords: stabilization, linear time-invariant systems, eigenvalue placement, quantifier elimination.

\section{Introduction}

This paper deals with linear time-invariant state-space systems. The essential conditions of controller design using state feedback in combination with an observer have been solved more than half a century ago $[20,27,32]$. The concepts of controllability and observability have been replaced by the weaker formulations of stabilizability and detectability in [21]. From a theoretical point of view, the design of a static output feedback controller is significantly more complicated [43]. The calculation of an appropriate gain matrix is still an area of active research $[15,16,22,36,45,55]$.

Formally, the existence of a static output feedback controller achieving prescribed design goals is a decision problem. The associated design requirements can be formulated as equations and inequalities over the real numbers. This type of decision problems can be solved using quantifier elimination. The theoretical foundations of this technique go back to Tarski's famous theorem published in 1948 [46]. Algorithmically, his approach was not applicable in practice. Starting around 1975,

\footnotetext{
${ }^{a}$ Institute of Control Theory, Technische Universität Dresden, 01062 Dresden, Germany, E-mail: klaus.roebenack@tu-dresden.de

${ }^{b} \mathrm{IAV} \mathrm{GmbH}$, Entwicklungszentrum Chemnitz/Stollberg, Germany E-mail: rick.vosswinkel@iav.de
} 
several promising algorithms and software tools for quantifier elimination have been developed $[6-9,13,51,53]$.

The application of quantifier elimination in control theory goes back to 1975 [3]. During the last decades, a few further applications have been discussed in the literature $[2,14,26]$. Due to the computational effort involved, quantifier elimination did not become a standard technique in controller design. However, modern algorithms and tools suggest that quantifier elimination may become more important for controller design in the near future. The authors will consider some scenarios of static output feedback design. This paper is an extended version of the results presented at [39]. Some results were also presented in German language in [40].

The paper is structured as follows. In Section 2, we recall well-known facts concerning state feedback design. The difficulties of static output feedback design are addressed in Section 3. The following Section 4 presents some background on quantifier elimination. The described methods are applied to the static output feedback design problem in Section 5. Finally, we will draw some conclusions in Section 6 .

\section{$2 \quad$ State Feedback Design}

In this section, we would like to recall some details concerning state feedback design. Consider a linear time-invariant state-space system

$$
\dot{x}=A x+B u, \quad y=C x
$$

with matrices $A \in \mathbb{R}^{n \times n}, B \in \mathbb{R}^{n \times m}$ and $C \in \mathbb{R}^{r \times n}$. The design of a controller is usually carried out via static state feedback

$$
u=-F x, \quad F \in \mathbb{R}^{m \times n}
$$

with a gain matrix $F$. This approach yields the closed-loop system

$$
\dot{x}=(A-B F) x .
$$

The eigenvalues of the closed-loop system (3) can be placed arbitrarily if and only if the system is controllable, i.e.,

$$
\forall s \in \mathbb{C}: \quad \operatorname{rank}(s I-A, B)=n .
$$

During the last decades, several design procedures such as Ackermann's formula have been developed and improved $[1,34,35]$.

If the system is not controllable, it may still be possible to achieve the most important goal of control, namely stabilization. The system (1) is called stabilizable if

$$
\exists F: \quad A-B F \text { is Hurwitz. }
$$

The system is stabilizable if and only if

$$
\forall s \in \mathbb{C}, \Re(s) \geq 0: \quad \operatorname{rank}(s I-A, B)=n,
$$


see [21]. Roughly speaking, condition (5) can be interpreted as the controllability of all unstable eigenvalues.

To compute a stabilizing feedback law (2), we consider the Lyapunov candidate function $V(x)=x^{T} P x$ with a symmetric positive definite matrix $P \succ 0$. The time derivative of $V$ along the dynamics of system (1) with the state feedback (2) reads

$$
\begin{aligned}
\dot{V}(x) & =\dot{x}^{T} P x+x^{T} P \dot{x} \\
& =x^{T}(A-B F)^{T} P x+x^{T} P(A-B F) x \\
& \stackrel{!}{=}-x^{T} Q x \quad \text { with } \quad Q \succ 0 .
\end{aligned}
$$

The last line demands negative definiteness of $\dot{V}$ corresponding to global exponential stability. The stability conditions resulting from (6) are fulfilled if and only if the Lyapunov equation

$$
A^{T} P+P A-F^{T} B^{T} P-P B F=-Q
$$

has a positive definite solution $P$ for an arbitrary positive definite matrix $Q$. Without loss of generality, we could use the identity matrix $Q:=I[37]$. Alternatively, we could formulate the stability condition in terms of a Lyapunov inequality

$$
A^{T} P+P A-F^{T} B^{T} P-P B F \prec 0 .
$$

For both $(7)$ and $(8)$ we seek a solution w.r.t. $(P, F)$. Due to the product terms $F^{T} B^{T} P$ and $P B F,(7)$ and (8) are bilinear. Therefore, linear solvers are not yet applicable.

The bilinearity in (7) and (8) cannot directly be removed because the matrix $B$ in the middle of the product is usually singular. Multiplying both sides of (8) with $W:=P^{-1}$ yields

$$
W A^{T}+A W-W F^{T} B^{T}-B F W \prec 0,
$$

where this inequality has to be solved w.r.t. $(W, F)$. The substitution $G=F W$ yields the linear matrix inequality (LMI)

$$
W A^{T}+A W-G^{T} B^{T}-B G \prec 0,
$$

which can numerically be solved w.r.t. $(W, G)$. From the re-substitution $F=$ $G W^{-1}$, we obtain the stabilizing gain matrix $F$.

In practice, the state feedback (2) is usually not directly implemented because not all components of the state are measured. In this case, the state is reconstructed using an observer

$$
\dot{\hat{x}}=A \hat{x}+B u+L(y-C \hat{x})
$$

with the gain matrix $L \in \mathbb{R}^{n \times r}$. Then, the estimated state $\hat{x}$ is feeded back with

$$
u=-F \hat{x}
$$

instead of (2). Existence and computation of the observer gain matrix $L$ are wellunderstood. However, the combination of (11) and (12) can be interpreted as a dynamic output feedback. 


\section{Static Output Feedback Design}

Now, we consider system (1) with the static output feedback

$$
u=-K y, \quad K \in \mathbb{R}^{m \times r} .
$$

From an implementation point of view, the static output feedback (13) is much simpler than the dynamic output feedback resulting from (11) and (12). The closedloop system

$$
\dot{x}=(A-B K C) x
$$

has the characteristic polynomial

$$
\mathrm{CP}(s)=\operatorname{det}(s I-(A-B K C))=a_{0}+a_{1} s+\cdots+a_{n-1} s^{n-1}+s^{n} .
$$

We want to assign new dynamics to the closed-loop system (14) described by the characteristic polynomial

$$
\mathrm{CP}^{*}(s)=a_{0}^{*}+a_{1}^{*} s+\cdots+a_{n-1}^{*} s^{n-1}+s^{n} .
$$

Unfortunately, the conditions for arbitrary eigenvalue placement via static output feedback are much more complicated compared to the static state feedback case. The necessary condition

$$
m r \geq n
$$

is straightforward. Sufficient conditions for generic systems have been discussed in $[12,23,28,50]$. After decades of research, the static output feedback eigenvalue placement was stated as an open problem in [43] and has been solved recently in $[15]$.

Why is the eigenvalue assignment problem so difficult? The determinant used to define the characteristic polynomial (15) is a multilinear functional. Therefore, the coefficients $a_{0}, \ldots, a_{n-1}$ may depend multilinearly on the entries of the closed-system matrix $A-B K C$ as well as the entries of the gain matrix $K$. As a consequence, the eigenvalue assignment problem

$$
\exists K \forall s: \quad \mathrm{CP}(s) \stackrel{!}{=} \mathrm{CP}^{*}(s)
$$

yields a multilinear system of equations, which may only have complex solutions.

For a generic system, the number of solutions is given by the Schubert number [5, $50]$

$$
d(m, r)=\frac{1 ! 2 ! \cdots(r-1) ! 1 ! 2 ! \cdots(m-1) !(m r) !}{1 ! 2 ! \cdots(m+r-1) !}=\frac{1 ! 2 ! \cdots(r-1) !(m r) !}{m ! \cdots(m+r-1) !} .
$$

An odd number of complex solutions would imply the existence of a real solution, which is required for the actual control implementation. The results on eigenvalue assignability for generic systems can be summarized as follows [50]: If $d(m, r)$ is odd, the eigenvalues of a generic system can be assigned arbitrarily by static output 
feedback (13) if and only if condition (17) holds. If $d(m, r)$ is even, the generic system has the arbitrary eigenvalue assignability if $m r>n$. For procedures to solve the assignment problem with static output feedback numerically see $[15,16,22,30,55]$ and references cited therein.

Instead of eigenvalue placement, we consider the stabilization problem now. The system is called stabilizable by static output feedback if

$$
\exists K: \quad A-B K C \text { is Hurwitz. }
$$

To derive conditions for stabilizability we consider the Lyapunov candidate function $V(x)=x^{T} P x$ with $P \succ 0$. Carrying out the procedure similarly to Section 2 yields

$$
P(A-B K C)+(A-B K C)^{T} P \prec 0 .
$$

The alternative formulation with $W:=P^{-1}$ reads

$$
(A-B K C) W+W(A-B K C)^{T} \prec 0 .
$$

For fixed $P$ or $W$, the inequalities $(20)$ and (21) are LMIs. In our application, we need to solve $(20)$ or $(21)$ simultaneously w.r.t. $(P, K)$ or $(W, K)$, respectively, which is difficult due to bilinearity. A simple substitution (as the transition form (9) to $(10)$ ) does not result in an equivalent formulation as the associated product terms $P B K C$ and $B K C W$ have an usually singular matrix between the factors $P, K$ or $W, K$, respectively. Approaches to resolve this bilinearity result in a non-convex optimization problem of coupled LMIs, see $[25,45]$. However, we have the possibility for solving this LMI iteratively, similar to the approaches summarized in [38] for a cooperativity-enforcing observer synthesis.

\section{Quantifier Elimination}

The conditions and properties introduced above can all be expressed using so-called prenex formulas. These prenex formulas can be described by

$$
G(Y, Z):=\left(\mathcal{Q}_{1} y_{1}\right) \cdots\left(\mathcal{Q}_{l} y_{l}\right) F(Y, Z)
$$

with $\mathcal{Q}_{i} \in\{\exists, \forall\}$ and the quantifier-free formula $F(Y, Z)$. A quantifier-free formula $F(Z)$ is given by a boolean combination of atomic formulas $f\left(z_{1}, \cdots, z_{k}\right)$ 。 0 with $\circ \in\{=,<,>, \leq, \geq, \neq\}$ and a polynomial $f\left(z_{1}, \ldots, z_{k}\right)$, using the operators $\vee, \wedge, \neg, \Longrightarrow$ and $\Longleftrightarrow$. From the control-theoretic point of view, we are in a first step interested in the solvability of the problems (4), (18) or (19) and in a second step we are looking for a specific solution. In other words, is there a possible control parameter configuration which solves the problem and how do we have to choose these parameters? Thus we are interested in a quantifier-free equivalent to the quantified conditions (4), (18) and (19). If such an equivalent exists or not is stated by the following theorem [4], which is a direct consequence of the Tarski-Seidenberg Theorem [44,46]: 
Theorem 1 (Quantifier Elimination Over The Real Closed Field). For every real prenex formula $G(Y, Z)$ there exists an equivalent quantifier-free formula $H(Z)$, i.e., for every $Y \in \mathbb{R}^{l}$ and $Z \in \mathbb{R}^{k}$ there holds

$$
G(Y, Z) \text { is true } \Longleftrightarrow H(Z) \text { is true. }
$$

To illustrate the idea of quantifier elimination let us consider the quadratic polynomial $f(x)=x^{2}+p x+q$. First, we might be interested in the parameter combinations for which at least one real root exists. This can be formulated with $\exists x: f(x)=0$, hence $Y=\{x\}$ and $Z=\{p, q\}$. An equivalent condition without quantifiers in the quantifier-free variables is $p^{2}-4 q \geq 0(\exists x: f(x)=0 \Longleftrightarrow$ $\left.p^{2}-4 q \geq 0\right)$. Secondly, let us consider the existence of two different real roots. A corresponding prenex formula is $\exists x, y: f(x)=0 \wedge f(y)=0 \wedge x \neq y$. The formulation $p^{2}-4 q>0$ comes up as a quantifier-free equivalent. Following this concept, $f(x)$ is positive definite if $p^{2}-4 q<0$ holds and the question, if there exists a configuration $(p, q)$ that $f(x)$ becomes negative definite $(\exists p, q \forall x: f(x)<0)$ leads to false. In the last case, all variables are quantified $(Y=\{x, p, q\} ; Z=\{\emptyset\})$. Thus, a decision problem has been derived and just true or false can result.

So the question arises if there exists a systematic approach to generate such quantifier-free equivalent formulations. There are several methods to achieve a quantifier elimination (QE). Historically the first algorithm was introduced by Tarski himself, but the computational complexity of this procedure cannot be bounded by any stack of exponentials. Nowadays, the three most common strategies are cylindrical algebraic decomposition (CAD), virtual substitution (VS) and real root classification techniques (RRC).

A quantifier-free formula given by a boolean combination of atomic formulas defines a semialgebraic set in $\mathbb{R}^{n}$. A crucial result of real algebraic geometry is, that the projection of a semialgebraic set from $\mathbb{R}^{n}$ to $\mathbb{R}^{n-1}$ is a semialgebraic set as well [4]. This idea is used by the first practically relevant algorithm, CAD [8]. Basically, this algorithm consists of three phases. In a first step, the semialgebraic set is successively projected to $\mathbb{R}^{1}$. The prenex formulas are afterward evaluated at the resulting semialgebraic sets in $\mathbb{R}^{1}$. In the end, the obtained results are lifted back to $\mathbb{R}^{n}$. Contrary to the other illustrated strategies, there exists no limitation w.r.t. the investigated polynomials. However, the computational load might increase double exponentially in the number of variables [11].

Better computational properties result with the virtual substitution $[31,51,52]$ and real root classification algorithms $[17,24,54]$. The method of virtual substitution is based on a formula equivalent to variable substitution using so-called elimination sets. This approach is only applicable to linear [51], quadratic [31] and cubic [52] polynomials, w.r.t. the quantified variables. Nevertheless, the computational complexity still grows exponentially in the number of quantified variables.

The term real root classification covers all approaches based on the number of real roots in a given interval. Utilizing Sturm-Habicht sequences, very effective algorithms could be achieved, especially for the sign definite conditions (SDC) $\forall x(x>0 \Longrightarrow f(x)=0)$. The computational effort of such a sign definite problem grows just exponential in the degree of the polynomials. 
Table 1: Quantifier elimination software and employed strategies

\begin{tabular}{|lllc|}
\hline Program & QE methods & Notes & References \\
\hline QEPCAD & CAD & Quantifier Elimination by & {$[9]$} \\
& & $\begin{array}{l}\text { Partial Cylindrical Algebraic } \\
\end{array}$ & \\
QEPCAD B & CAD & Decomposition & \\
Redlog & CAD, VS & Reduce package & {$[6]$} \\
SyNRAC & CAD, VS, SDC & Maple toolbox & {$[13]$} \\
RegularChains & CAD & Maple toolbox & {$[24,53]$} \\
\hline
\end{tabular}

During the last two decades, some powerful software tools to handle QE problems have been developed. A basic overview of these tools and the employed QE strategies is given in Table 1.

\section{$5 \quad$ Stabilizability and Stabilization}

\subsection{Stabilization based on Routh, Hurwitz etc.}

To the authors' knowledge, stabilizability and stabilization by static output feedback was the first application of QE in control theory [3]. Consider system (1) with the static output feedback (13). The stabilizability problem (19) can be stated as follows:

Proposition 1 (Stabilizability). System (1) is stabilizable by a static output feedback (13) if and only if

$$
\exists k_{11} \cdots \exists k_{m r}: \quad \mathrm{CP}(\cdot) \text { is a Hurwitz polynomial. }
$$

This is essentially a reformulation of (19) in more direct terms of a prenex formula. The Hurwitz property of the characteristic polynomial (15) (i.e., all roots have negative real parts) can be verified using the Routh, Hurwitz, or LiénardChipart test:

$$
\begin{aligned}
& n=2: a_{0}>0 \wedge a_{1}>0, \\
& n=3: a_{0}>0 \wedge a_{1}>0 \wedge a_{1} a_{2}-a_{0}>0, \\
& n=4: a_{0}>0 \wedge a_{1}>0 \wedge a_{2}>0 \wedge a_{1} a_{2} a_{3}-a_{1}^{2}-a_{0} a_{3}^{2}>0 .
\end{aligned}
$$

Based on the formulas (23) and (24), the stabilizability of the system can formally be verified using QE. In [3], the approach was illustrated on a system with $n=3$, $m=1$ and $r=2$, where QE was carried out by hand. The same example was considered in [45] using QEPCAD [9] and in [42] using REDLOG [13]. 
Example 1. We consider the system

$$
\begin{aligned}
A & =\left(\begin{array}{cccc}
-1 & 0 & 0 & 0 \\
0 & -2 & 0 & 0 \\
0 & 0 & 1 & 0 \\
0 & 0 & 0 & 2
\end{array}\right), \quad B=\left(\begin{array}{ll}
1 & 0 \\
1 & 0 \\
1 & 1 \\
1 & 0
\end{array}\right), \\
C & =\left(\begin{array}{llll}
1 & 1 & 1 & 1 \\
0 & 0 & 0 & 1
\end{array}\right), \quad K=\left(\begin{array}{ll}
k_{11} & k_{12} \\
k_{21} & k_{22}
\end{array}\right) .
\end{aligned}
$$

derived from from [55, Example 4.1] having $n=4$ and $m=r=2$. The coefficients of the characteristic polynomial (15) are

$$
\begin{aligned}
& a_{0}=-2 k_{11} k_{22}+2 k_{12} k_{21}-4 k_{21}-2 k_{12}+4, \\
& a_{1}=3 k_{11} k_{22}-3 k_{12} k_{21}+4 k_{21}+k_{12}+10 k_{11}, \\
& a_{2}=-k_{11} k_{22}+k_{12} k_{21}+k_{21}+2 k_{12}-5, \\
& a_{3}=-k_{21}-k_{12}-4 k_{11} .
\end{aligned}
$$

To verify the stabilizability based on (23) with (24) we used the computer algebra system REDUCE with the package REDLOG [13]. For the computations we employed a PC with Intel ${ }^{\circledR}$ Core $^{\mathrm{TM}}$ i5-6500 CPU at $3.2 \mathrm{GHz}$ and $64 \mathrm{GiB}$ RAM under the Linux system Fedora 30 (64 bit). For QE we used the virtual substitution method from [29] (i.e., the function rlqe with the switch on of sfvs). For system (25), eliminating the existence quantifiers from (23) with (24) took approximately $430 \mathrm{~ms}$ resulting in the quantifier-free formula true. Hence, the system is stabilizable by static output feedback. Note that if we replace the system matrix $A$ by $-A$, the resulting system is not stabilizable by static output feedback. These results are in accordance with [55]. We made the source files available on Github [56].

Remark 1. Applying QE to (23), we can formally verify the solvability of the stabilization problem (19). If the stabilization problem is solvable, we have a justification to apply numerical methods to compute the gain $K$. Moreover, we could also employ QE directly to compute a stabilizing gain $K=\left(k_{i j}\right)$ step-by-step:

1. Omit the existence quantifier for one variable $k_{i j}$,

2. Compute the feasible set,

3. Specify the variable and proceed with the next variable.

An entry $k_{i j}$ not associated with a quantifier becomes a free variable. QE applied to (23) yields a quantifier-free formula in this (single) variable $k_{i j}$ (assuming no further parameters are involved).

Example 2. Now, we want to compute a stabilizing gain matrix $K$ for system (25) from Example 1. Omitting the existence quantifier for $k_{11}$ yields true, i.e., we can select any real value. Here $k_{11}:=0$ is chosen. Afterward, we omit the quantifier 
of $k_{12}$ and QE yields $k_{12}>2$. We set $k_{12}:=3$ and proceed with $k_{21}$ resulting in $k_{21}>1$. With $k_{21}:=2$, the characteristic polynomial does not depend anymore on $k_{22}$. With the choice $k_{22}:=0$ the gain matrix has the form

$$
K=\left(\begin{array}{ll}
0 & 3 \\
2 & 0
\end{array}\right)
$$

and yields the eigenvalues $s_{1}=s_{2}=s_{3}=-1$ and $s_{4}=-2$ of the closed-loop system (14). Alternatively, we could set $k_{11}:=1$ in the first step. In the second step regarding $k_{12}$ we obtain true and set $k_{12}:=0$. QE in the third step also yields true, where we would set $k_{21}:=0$. This results in the conditions that $k_{22}$ must be smaller than the smallest real root of the polynomial $\phi\left(k_{22}\right)=k_{22}^{2}+24 k_{22}+12$. The quadratic polynomial has the roots $k_{22}=-12 \pm 2 \sqrt{33} \approx\{-0.511,-23.489\}$. The conditions above are fulfilled for $k_{22}=-24$. With the gain matrix

$$
K=\left(\begin{array}{cc}
1 & 0 \\
0 & -24
\end{array}\right),
$$

the closed-loop system has the eigenvalues $s_{1,2} \approx-0.011 \pm 3.95 j, s_{3} \approx-1.205$ and $s_{4} \approx-2.773$. Both computed eigenvalue constellations stabilize the system.

Example 3. The example system

$$
A=\left(\begin{array}{llll}
0 & 0 & 0 & 0 \\
0 & 0 & 0 & 0 \\
2 & 0 & 1 & 2 \\
0 & 2 & 2 & 1
\end{array}\right), \quad B=\left(\begin{array}{ll}
1 & 0 \\
0 & 1 \\
0 & 0 \\
0 & 0
\end{array}\right), \quad C=\left(\begin{array}{cccc}
4 & -3 & 3 & 0 \\
-2 & 2 & -1 & 2
\end{array}\right)
$$

was introduced in [18]. In [10], the authors considered different static output feedback structures

$$
K=\left(\begin{array}{ll}
k_{11} & k_{12} \\
k_{21} & k_{22}
\end{array}\right), \quad K=\left(\begin{array}{cc}
k_{11} & 0 \\
k_{21} & k_{22}
\end{array}\right), \quad K=\left(\begin{array}{cc}
k_{11} & 0 \\
0 & k_{22}
\end{array}\right)
$$

for system (29). The verification of stabilizability by these gain matrices using Proposition 1 required approximately $5 \mathrm{~min} 50 \mathrm{~s}, 0.95 \mathrm{~s}$ or $0.36 \mathrm{~s}$ computation time, respectively. Now, let us consider the diagonal matrix in Eqs. (30) (right). With $k_{11}$ as a free variable and $k_{22}$ as a quantified variable, QE yields the condition $k_{11}>7 / 3=2 . \overline{3}$. Conversely, if we select $k_{22}$ as a free and $k_{11}$ as a quantified variable, QE returns the result that $k_{22}$ must be greater than the largest real root of the polynomial $\phi\left(k_{22}\right)=9 k_{22}^{2}-46 k_{22}+9$. The quadratic polynomial has the roots $k_{22}=(23 \pm 8 \sqrt{7}) / 9 \approx\{0.204,4.907\}$, i.e., $k_{22} \gtrsim 4.907$. If we select one of these variables according to the specified bounds, then there is a value for the other variable stabilizing the system.

Remark 2 (Robust stabilization). Let $s_{0}<0$ be a stability margin, i.e., a distance to the imaginary axis. Applying the substitution $s \mapsto s-s_{0}$ to the characteristic polynomial (15), the corresponding conditions (24) ensure that any root $s_{i} \in \mathbb{C}$ of (14) fulfills $\Re\left(s_{i}\right)<s_{0}$. In a similar manner we can define two bounds $\underline{s}_{0}, \bar{s}_{0}$ with $\underline{s}_{0}<\bar{s}_{0}<0$ such that all eigenvalues fulfill $\underline{s}_{0} \leq \Re\left(s_{i}\right)<\bar{s}_{0}$. 


\subsection{Stabilization based on Lyapunov formulations}

As an alternative to the approach based on the characteristic polynomial presented above, we may directly use the Lyapunov condition (20) or $(21)[47,49]$. This leads to the prenex formulation

$$
\exists P, K: \quad P \succ 0 \wedge P(A-B K C)+(A-B K C)^{T} P \prec 0 .
$$

From a computational point of view the equivalent equality condition

$$
\exists P, K: \quad P \succ 0 \wedge \underbrace{P(A-B K C)+(A-B K C)^{T} P+I}_{M:=}=0
$$

is advantageous. The prenex formulation (32) leads to $n(n+1) / 2$ equality conditions and based on Sylvester's the leading principal criterion [33] it turns into $n$ inequality conditions for the definiteness of $P$ :

$$
P \succ 0 \quad \Longleftrightarrow \quad p_{11}>0 \wedge \operatorname{det}\left(\begin{array}{ll}
p_{11} & p_{12} \\
p_{21} & p_{22}
\end{array}\right)>0 \wedge \ldots \wedge \operatorname{det}(P)>0 .
$$

The resulting equality constraints of the Lyapunov equation in (32) are bilinear in the parameters $p_{11}, \ldots, p_{n n}, k_{11}, \ldots, k_{m r}$. In contrast to (32), the formulation (31) leads to $n$ additional inequality conditions instead of $n(n+1) / 2$ equality conditions due to the leading principal criterion, i.e., condition (31) results over all in $2 n$ inequality constraints. However, these conditions may result in higher order monoms in the parameters $p_{11}, \ldots, p_{n n}, k_{11}, \ldots, k_{m r}$ with increasing dimensions of the principal minors. These high order polynomial conditions result in demanding computational effort. This especially holds true for increasing systems dimension. These considerations are summed up by the following proposition.

Proposition 2 (Stabilizability with the Lyapunov equation). System (1) is stabilizable by a static output feedback (13) if and only if

$$
\exists p_{11}, \ldots, p_{n n}, k_{11}, \ldots, k_{m r}: m_{11}=0 \wedge \cdots \wedge m_{n n}=0 \wedge\left|P_{1}\right|>0 \wedge \cdots \wedge\left|P_{n}\right|>0
$$

with $m_{i j}$ being the element of the $i$-th row and the $j$-th column of the matrix $M$ defined in (32) and $\left|P_{i}\right|$ denoting the $i$-th leading principal minor of $P$.

Remark 3 (Robust stabilization with the Lyapunov equation). Equivalently to Remark 2, a robust stabilization can be achieved by replacing the matrix $M$ defined in (32) with

$$
M:=P\left(A-s_{0} I-B K C\right)+\left(A-s_{0} I-B K C\right)^{T} P+I .
$$

This substitution shifts the stability condition to a line parallel to the imaginary axis through the point $s_{0}<0$ on the real axis. 


\subsection{Eigenvalue Placement, Real and Interval Stabilization}

Consider the question of arbitrary eigenvalue placement (real or complex conjugate pairs) via static output feedback. This problem is equivalent to the question, whether any polynomial (16) with real coefficients $a_{0}^{*}, \ldots, a_{n-1}^{*} \in \mathbb{R}$ can be imposed to the closed-loop system by output feedback (13). This leads directly to the following formulation $[3,41]$ :

Proposition 3 (Arbitrary Eigenvalue Placement). Any characteristic polynomial (16) can be assigned to the characteristic polynomial (15) of the closed-loop system (14) if and only if

$$
\forall a_{0}^{*} \cdots \forall a_{n-1}^{*} \exists k_{11} \ldots \exists k_{m r}: \quad a_{0}=a_{0}^{*} \wedge \ldots \wedge a_{n-1}=a_{n-1}^{*} .
$$

The comparison between (15) and (16) as in (18) is carried out by coefficient matching.

Example 4. We continue with system (25) from Example 1. Carrying out QE for the associated condition (34) yields false, i.e., the eigenvalues cannot be assigned arbitrarily. The REDUCE script required approximately $120 \mathrm{~ms}$ computation time, from which QE took about $20 \mathrm{~ms}$.

An arbitrary eigenvalue placement is not absolutely necessary in practice. Normally, one has a specific idea of the eigenvalues to be imposed.

Proposition 4 (Specific Eigenvalue Placement). Consider a given prescribed characteristic polynomial (16). This polynomial can be imposed to the system's characteristic polynomial (15) by static output feedback (13) if and only if

$$
\exists k_{11} \ldots \exists k_{m r}: \quad a_{0}=a_{0}^{*} \wedge \ldots \wedge a_{n-1}=a_{n-1}^{*} .
$$

Example 5. Consider system (25) from Example 1. First, we try to place all eigenvalues at $s_{1, \ldots, 4}=-1$, i.e., $\mathrm{CP}^{*}(s)=(s+1)^{4}$. QE applied to (35) results in false, i.e., this specific eigenvalue assignment is not possible. Second, we want to place the eigenvalues at $-1,-2,-3,-4$, i.e., $\mathrm{CP}^{*}(s)=(s+1)(s+2)(s+3)(s+4)$. The elimination of the quantifier occurring in (35) yields true. The calculation of the entries of the gain matrix as described in Remark 1 results in

$$
K=\left(\begin{array}{ll}
0 & 5 \\
5 & *
\end{array}\right)
$$

with an arbitrary entry for $k_{22}$.

With the described approach we can place eigenvalues at prescribed positions, or obtain the information, that the desired placement is not possible. For stabilization, these eigenvalues must be placed in the open complex left half plane. The procedure described in Sections 5.1 and 5.2 may yield non-real eigenvalues occurring as complex conjugate pairs. Such an eigenvalue constellation corresponds to attenuated oscillations in the time domain, that may not be desired. If we want to 
avoid these oscillations, we could aim for a real stabilization. This can be seen as an eigenvalue placement problem, where the desired characteristic polynomial has the form

$$
\mathrm{CP}^{*}(s)=\left(s-s_{1}\right) \cdots\left(s-s_{n}\right) \quad \text { with } \quad s_{1}, \ldots, s_{n}<0 .
$$

Proposition 5 (Real Stabilizability I). System (1) is stabilizable by static output feedback (13) such that the closed-loop system (14) has only real eigenvalues if and only if

$$
\exists k_{11} \ldots \exists k_{m r} \exists s_{1} \cdots \exists s_{n}: s_{1}<0 \wedge \ldots s_{n}<0 \wedge a_{0}=a_{0}^{*} \wedge \ldots \wedge a_{n-1}=a_{n-1}^{*}
$$

with a polynomial (16) of the form (36).

Compared to (35), the prenex formula (37) contains $n$ more quantified variables. However, the formulation (36) could be used to describe other design goals, which are summarized below:

1. Real stabilization: $s_{1}<0, \ldots, s_{n}<0$,

2. Robust real stabilization: $s_{1} \leq s_{0}, \ldots, s_{n} \leq s_{0}$ with $s_{0}<0$,

3. Interval assignment: $s_{1} \in \mathcal{I}_{1}, \ldots, s_{n} \in \mathcal{I}_{n}$, where $\mathcal{I}_{1}, \ldots, \mathcal{I}_{n} \subseteq(-\infty, 0)$ can be open, half-open or closed intervals.

Furthermore, any combination thereof is possible as well. In particular, the individual interval assignment offers a possibility to relax a desired but not achievable design goal in eigenvalue placement.

Example 6. For system (25) from Example 1 we want to achieve a real stabilization with $s_{1}<0, \ldots, s_{4}<0$. The solvability of the assignment problem with a full $2 \times 2$ matrix $K$ could easily be verified using QE. Unfortunately, we were not able to carry out the stepwise computation of the entries as described in Remark 1. Clearly, the significant computational effort is due to the numbers of variables and quantifiers. As shown in (27) and (28), stabilization by static output feedback can be achieved with gain matrices of the structures

$$
K=\left(\begin{array}{cc}
0 & k_{12} \\
k_{21} & 0
\end{array}\right) \quad \text { or } \quad K=\left(\begin{array}{cc}
k_{11} & 0 \\
0 & k_{22}
\end{array}\right) .
$$

The solvability of the above mentioned design goal could be verified for the structures (38) with QE in about $1.2 \mathrm{~s}$ and $3.4 \mathrm{~s}$, respectively. The matrix (27) with the first structure already achieved our design goal. Using $k_{12}$ and $k_{21}$ as free variables, quantifier elimination results in the condition $k_{12} \geq-3 \wedge k_{21} \geq 2$ for the aforementioned robust real eigenvalue placement goal. However, the diagonal matrix (28) stabilized the system, but violated the goals stated now (complex conjugate pair instead of purely real eigenvalues). With $k_{11}$ or $k_{22}$ as a free variable, the computation with REDLOG exceeds the RAM available. 
The approach for an arbitrary interval placement allows a very specific eigenvalue placement, but increases the computational effort significantly due to the additional quantified variables $s_{1}, \ldots, s_{n}$. In purely formal terms, the algorithms will deliver a result in finite time, but the specific duration may not be acceptable. Alternatively, the number of real roots in an interval can be computed using Sturm or Sturm-Habicht sequences $[19,40]$. In particular, real stabilization can be seen as an eigenvalue placement into the interval $(-\infty, 0)$.

Proposition 6 (Real Stabilizability II). System (1) is stabilizable by static output feedback (13) such that the closed-loop system (14) has only real eigenvalues if and only if

$$
\begin{aligned}
& \exists k_{11} \cdots \exists k_{m r}: \\
& n=2: \quad a_{0}>0 \wedge a_{1}>0 \wedge a_{1}^{2}-4 a_{0}>0, \\
& n=3: \quad a_{0}>0 \wedge a_{1}>0 \wedge 9 a_{0}-a_{1} a_{2}<0 \wedge \\
& \quad 27 a_{0}^{2}-18 a_{0} a_{1} a_{2}+4 a_{0} a_{2}^{3}+4 a_{1}^{3}-a_{1}^{2} a_{2}^{2}<0 .
\end{aligned}
$$

For higher order systems, the appropriate conditions become more complicated, but can be generated automatically. The formulation is similar to that in Proposition 1 .

Example 7. We continue with the system from Example 6 with the diagonal matrix shown in (38). Using $k_{11}$ as free variable and the formulation as in Proposition 6, QE results in the condition that $k_{11}$ must be larger than the largest real root of the polynomial $\phi_{1}\left(k_{11}\right)=673280 k_{11}^{6}-3606912 k_{11}^{5}+1743888 k_{11}^{4}-2483712 k_{11}^{3}+$ $1259880 k_{11}^{2}-338616 k_{11}-42875$, i.e., $k_{11} \gtrsim 4.97$. We set $k_{11}:=5$. For $k_{22}$ we obtain the condition that this entry should be smaller than the smallest real root of the polynomial $\phi_{2}\left(k_{22}\right)=42875 k_{22}^{6}+5229396 k_{22}^{5}+76959408 k_{22}^{4}-622283328 k_{22}^{3}+$ $14829211248 k_{22}^{2}+18901165248 k_{22}+10690284160$, i.e., $k_{22} \lesssim-28.93$ Therefore, we obtain the gain matrix

$$
K=\left(\begin{array}{cc}
5 & 0 \\
0 & -29
\end{array}\right)
$$

resulting in the closed loop eigenvalues $s_{1} \approx-1.209, s_{2} \approx-5.791, s_{3}=-6$ and $s_{4}=-7$, i.e., our design goal is fulfilled.

Example 8. We want to achieve the real stabilization of system (29) from Example 3 with the gain matrix (30) in diagonal form. QE results in the condition that $k_{11}$ must be larger than the largest real root of $\phi_{1}\left(k_{11}\right)=25 k_{11}^{4}-$ $774 k_{11}^{3}-203 k_{11}^{2}-5292 k_{11}+1372$, i.e., $k_{11} \gtrsim 30.258$. Without fixing $k_{11}$, we use $k_{22}$ as a free variable. The entry $k_{22}$ must be larger than the largest real root of $\phi_{2}\left(k_{22}\right)=25 k_{22}^{4}-1452 k_{22}^{3}-12426 k_{22}^{2}-13228 k_{22}+5913$, i.e., $k_{22} \gtrsim 65.760$. As in Example 3 these limitations in the parameter space are obtained if we consider the variables $k_{11}$ and $k_{22}$ independently. The values of $\left(k_{11}, k_{22}\right)$, for which stability and real stability are achieved, are plotted numerically in Fig. 1. The boundaries between the different areas correspond to the values computed in Example 3 and 8. Alternatively, these sets can be calculated using parameter space methods, see $[22,48]$ and references cited there. 


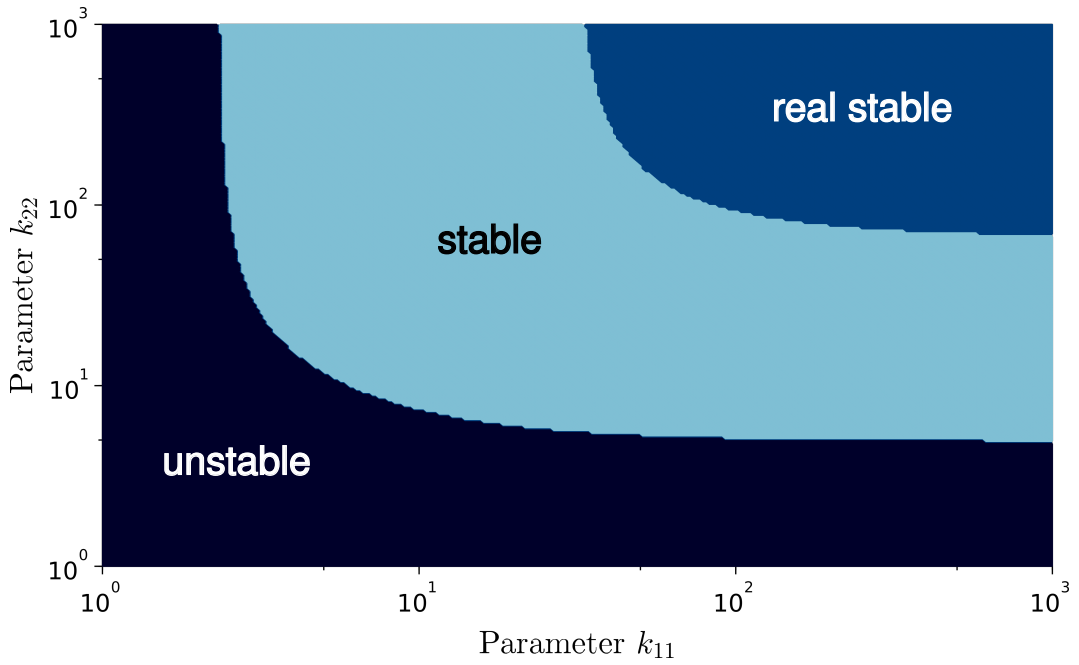

Figure 1: Stability properties of system (29) of Examples 3 and 8 plotted in the parameter space

\section{Conclusions}

We showed that static output feedback design with several constraints can be carried out using quantifier elimination. Due to modern algorithms such as virtual substitution, it is to be expected that this approach will gain in importance in the near future. Furthermore, parameter uncertainties and other design parameters can directly be addressed. Nevertheless, the inherent computational complexity currently prevents the applicability to high order systems. However, a further increase in processing power and algorithmic development will help to overcome these limitations. In particular, the extent to which the QE methods can be improved by massive parallelisation should be investigated on an algorithmic level.

\section{References}

[1] Ackermann, J. E. On the synthesis of linear control systems with specified characteristics. Automatica, 13(1):89-94, January 1977. DOI: 10.1016/0005-1098(77)90012-7.

[2] Anai, H. and Hara, S. A parameter space approach to fixed-order robust controller synthesis by quantifier elimination. Int. J. Control, 79(11):13211330, 2006. DOI: 10.1080/00207170600726550. 
[3] Anderson, B., Bose, N., and Jury, E. Output feedback stabilization and related problems-solution via decision methods. IEEE Trans. on Automatic Control, 20(1):53-66, February 1975. DOI: 10.1109/TAC.1975.1100846.

[4] Basu, S., Pollack, R., and Roy, M.-F. Algorithms in Real Algebraic Geometry. Springer, Berlin, Heidelberg, 2 edition, 2006.

[5] Brockett, R. and Byrnes, C. Multivariable Nyquist criteria, root loci, and pole placement: A geometric viewpoint. IEEE Transactions on Automatic Control, 26(1):271-284, 1981. DOI: 10.1109/TAC.1981.1102571.

[6] Brown, C. W. QEPCAD B: A program for computing with semi-algebraic sets using CADs. ACM SIGSAM Bulletin, 37(4):97-108, 2003. DOI: $10.1145 / 968708.968710$.

[7] Chen, C. and Maza, M. M. Real quantifier elimination in the RegularChains library. In Hong, H. and Yap, C., editors, Mathematical Software - IMCS2014, volume 8295 of Lecture Notes in Computer Science, pages 283-290, Berlin, Heidelberg, 2014. Springer-Verlag.

[8] Collins, G. E. Quantifier elimination for real closed fields by cylindrical algebraic decompostion. In Automata Theory and Formal Languages 2nd GI Conference Kaiserslautern, May 20-23, 1975, pages 134-183. Springer, 1975.

[9] Collins, G. E. and Hong, H. Partial cylindrical algebraic decomposition for quantifier elimination. Journal of Symbolic Computation, 12(3):299-328, 1991. DOI: $10.1016 /$ S0747-7171(08)80152-6.

[10] Crusius, C. A. R. and Trofino, A. Sufficient LMI conditions for output feedback control problems. IEEE Transactions on Automatic Control, 44(5):1053-1057, 1999. DOI: $10.1109 / 9.763227$.

[11] Davenport, J. H. and Heintz, J. Real quantifier elimination is doubly exponential. Journal of Symbolic Computation, 5(1):29-35, 1988. DOI: 10.1016/S0747-7171(88)80004-X.

[12] Davison, E. J. and Wang, S. H. On pole assignment in linear multivariable systems using output feedback. IEEE Trans. on Automatic Control, 20(4):516518, 1975. DOI: 10.1109/TAC.1975.1101023.

[13] Dolzmann, A. and Sturm, T. Redlog: Computer algebra meets computer logic. ACM SIGSAM Bulletin, 31(2):2-9, 1997. DOI: 10.1145/261320.261324.

[14] Dorato, P. Quantified multivariable polynomial inequalities - The mathematics of practical control design problems. IEEE Control Systems Magazine, 20(5):48-58, October 2000. DOI: 10.1109/37.872903.

[15] Franke, M. Eigenvalue assignment by static output feedback - on a new solvability condition and the computation of low gain feedback matrices. Int. J. Control, 87(1):64-75, 2014. DOI: 10.1080/00207179.2013.822102. 
[16] Franke, M. and Röbenack, K. Calculation of constant output feedback matrices for pole placement by a Gauss-Newton method. Control and Intelligent Systems, 42(3):225-230, 2014. DOI: 10.2316/Journal.201.2014.3.201-2579.

[17] Gonzalez-Vega, L., Lombardi, H., Recio, T., and Roy, M.-F. Sturm-Habicht sequence. In Proc. of the ACM-SIGSAM 1989 International Symposium on Symbolic and Algebraic Computation, pages 136-146, Portland, Oregon, USA, 1989. DOI: $10.1145 / 74540.74558$.

[18] Gu, Guoxiang. On the existence of linear optimal control with output feedback. SIAM Journal on control and Optimization, 28(3):711-719, 1990. DOI: $10.1137 / 0328041$.

[19] Habicht, W. Eine Verallgemeinerung des Sturmschen Wurzelzählverfahrens. Commentarii Mathematici Helvetici, 21(1):99-116, December 1948. DOI: 10.1007/BF02568028.

[20] Hautus, M. L. J. Controllability and observability conditions for linear autonomous systems. Ned. Akad. Wetenschappen, Proc. Ser. A, 72:443-448, 1969.

[21] Hautus, M. L. J. Stabilization, controllability, and observability for linear autonomous systems. Ned. Akad. Wetenschappen, Proc. Ser. A, 73:448-455, 1970.

[22] Henrion, D. and Sebek, M. Plane geometry and convexity of polynomial stability regions. In Proc. Int. Symposium on Symbolic and Algebraic Computation, pages 111-116, 2008. DOI: 10.1145/1390768.1390786.

[23] Hermann, R. and Martin, C. Applications of algebraic geometry to systems theory. Part I. IEEE Transactions on Automatic Control, 22(1):19-25, 1977. DOI: $10.1109 /$ TAC.1977.1101395.

[24] Iwane, H., Yanami, H., Anai, H., and Yokoyama, K. An effective implementation of symbolic-numeric cylindrical algebraic decomposition for quantifier elimination. Theoretical Computer Science, 479:43-69, 2013. DOI: $10.1016 / j . t c s .2012 .10 .020$.

[25] Iwasaki, T. and Skelton, R. E. Parametrization of all stabilizing controllers via quadratic Lyapunov functions. Journal of Optimization Theory and Applications, 85(2):291-307, 1995. DOI: 10.1007/BF02192228.

[26] Jirstrand, M. Nonlinear control system design by quantifier elimination. Journal of Symbolic Computation, 24(2):137-152, August 1997. DOI: 10.1006/jsco.1997.0119.

[27] Kalman, R. E. On the general theory of control systems. In First International Congress of the International Federation of Automatic Control (IFAC), Moscow, 1960. 
[28] Kimura, H. On pole assignment by output feedback. International Journal of Control, 28(1):11-22, 1978. DOI: 10.1080/00207177808922432.

[29] Košta, M. New Concepts for Real Quantifier Elimination by Virtual Substitution. Dissertation, Universität des Saarlandes, Fakultät für Mathematik und Informatik, Saarbrücken, Germany, 2016. https://publikationen.sulb. uni-saarland.de/handle/20.500.11880/26735.

[30] Lee, T. H., Wang, Q. G., and Koh, E. K. An iterative algorithm for pole placement by output feedback. IEEE Trans. on Automatic Control, 39(3):565568, March 1994. DOI: 10.1109/9.280760.

[31] Loos, R. and Weispfenning, V. Applying linear quantifier elimination. The Computer Journal, 36(5):450-462, 1993. DOI: 10.1093/comjnl/36.5.450.

[32] Luenberger, D. G. Observing the state of a linear system. IEEE Trans. Mil. Electronics, ME-8(2):74-80, 1964. DOI: 10.1109/TME.1964.4323124.

[33] Marcus, M. and Minc, H. A Survey of Matrix Theory and Matrix Inequalities. Dover, 1992.

[34] Mehrmann, V. and Xu, H. An analysis of the pole placement problem. I. The single-input case. Electronic Transactions on Numerical Analysis, 4:89-105, September 1996. http://eudml.org/doc/119479.

[35] Mehrmann, V. and Xu, H. An analysis of the pole placement problem. II. The multi-input case. Electronic Transactions on Numerical Analysis, 5:77-97, September 1997. http://eudml.org/doc/119610.

[36] Parlakçi, A. Design of a static output feedback H-infinity controller for linear time-invariant systems: An LMI approach. In International Conference on System Theory, Control and Computing (ICSTCC 2018), Sinaia, Romania, October 2018. DOI: 10.1109/ICSTCC.2018.8540651.

[37] Patel, R. V. and Toda, M. Quantitative measures of robustness for multivariable systems. In Joint Automatic Control Conference, 1980. DOI: 10.1109/JACC. 1980.4232116.

[38] Rauh, A., Kersten, J., and Aschemann, H. Linear matrix inequality techniques for the optimization of interval observers for spatially distributed heating systems. In IEEE International Conference on Methods 8 Models in Automation Es Robotics (MMAR), pages 138-143, Miedzyzdroje, Poland, 2018. DOI: 10.1109/MMAR. 2018.8486120.

[39] Röbenack, K. and Voßwinkel, R. Static output feedback control by interval eigenvalue placement using quantifier elimination. In 11th Summer Workshop on Interval Methods (SWIM 2018), Rostock, Germany, 2018. 
[40] Röbenack, K. and Voßwinkel, R. Lösung regelungstechnischer Problemstellungen mittels Quantorenelimination. Automatisierungstechnik, 2019. DOI: $10.1515 /$ auto-2019-0045.

[41] Röbenack, K., Voßwinkel, R., and Franke, M. On the eigenvalue placement by static output feedback via quantifier elimination. In Mediterranean Conference on Control and Automation (MED'18), pages 133-138, Zadar, Croatia, June 2018. DOI: $10.1109 /$ MED. 2018.8442817.

[42] Röbenack, K., Voßwinkel, R., Franke, M., and Franke, M. Stabilization by static output feedback: A quantifier elimination approach. In International Conference on System Theory, Control and Computing (ICSTCC 2018), pages 715-721, Sinaia, Romania, October 2018. DOI: 10.1109/ICSTCC. 2018.8540735 .

[43] Rosenthal, J. and Willems, J. Open problems in the area of pole placement. In Blondel, Vincent D., Sontag, Eduardo D., Vidyasagar, M., and Willems, Jan C., editors, Open Problems in Mathematical Systems and Control Theory, Communication and Control Engineering Series, pages 181-191, London, 1999. Springer-Verlag.

[44] Seidenberg, A. A new decision method for elementary algebra. Annals of Mathematics, 60(2):365-374, 1954. DOI: 10.2307/1969640.

[45] Syrmos, V. L., Abdallah, C. T., Dorato, P., and Grigoriadis, K. Static output feedback - A survey. Automatica, 33(2):125-137, 1997. DOI: 10.1016/S0005-1098(96) 00141-0.

[46] Tarski, A. A Decision Method for a Elementary Algebra and Geometry. Project rand. Rand Corporation, 1948.

[47] Tong, J. and Bajcinca, N. Computation of feasible parametric regions for Lyapunov functions. In Asian Control Conference (ASCC), pages 2453-2458, 2017. DOI: $10.1109 /$ ASCC. 2017.8287559.

[48] Voßwinkel, R., Pyta, L., Schrödel, F., Mutlu, İ., Mihailescu-Stoica, D., and Bajcinca, N. Performance boundary mapping for continuous and discrete time linear systems. Automatica, 107:272-280, 2019. DOI: 10.1016/j. automatica. 2019.05.055.

[49] Voßwinkel, R., Tong, J., Röbenack, K., and Bajcinca, N. Stability bounds for systems and mechanisms in linear descriptor form. In $59^{\text {th }}$ Ilmenau Scientific Colloquium, September 2017. https://nbn-resolving.org/urn:nbn: de:gbv:ilm1-2017iwk-115:1.

[50] Wang, X. A. Grassmannian, central projection, and output feedback pole assignment of linear systems. IEEE Trans. on Automatic Control, 41(6):786794, June 1996. DOI: 10.1109/9.506231. 
[51] Weispfenning, V. The complexity of linear problems in fields. Journal of Symbolic Computation, 5(1-2):3-27, $1988 . \quad$ DOI: 10.1016/S0747-7171(88)80003-8.

[52] Weispfenning, V. Quantifier elimination for real algebra - the cubic case. In Proc. of the Int. Symp. on Symbolic and Algebraic Computation (ISSAC), pages 258-263, 1994. DOI: 10.1145/190347.190425.

[53] Yanami, H. and Anai, H. The Maple package SyNRAC and its application to robust control design. Future Generation Computer Systems, 23(5):721-726, 2007. DOI: $10.1016 /$ j.future.2006.10.009.

[54] Yang, L., Hou, X., and Zeng, Z. Complete discrimination system for polynomials. Science in China Series E Technological Sciences, 39(6):628646, 1996.

[55] Yannakoudakis, A. G. The static output feedback from the invariant point of view. IMA Journal of Mathematical Control and Information, 33(3):639-668, 2016. DOI: 10.1093/imamci/dnu057.

[56] Static output feedback (REDUCE source files). https://github.com/ TUD-RST/static-output-feedback. 